\title{
Likely Sexual Transmission of Zika Virus from a Man with No Symptoms of Infection — Maryland, 2016
}

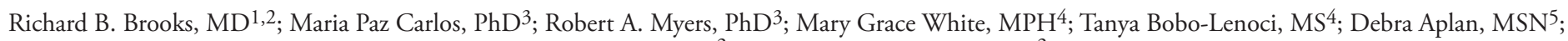
David Blythe, $\mathrm{MD}^{2}$; Katherine A. Feldman, $\mathrm{DVM}^{2}$

On August 26, 2016, this report was posted as an MMWR Early Release on the MMWR website (http://www.cdc.gov/mmwr).

In June 2016, the Maryland Department of Health and Mental Hygiene (DHMH) was notified of a nonpregnant woman who sought treatment for a subjective fever and an itchy rash, which was described as maculopapular by her provider. Laboratory testing at the Maryland DHMH Laboratories Administration confirmed Zika virus infection. Case investigation revealed that the woman had not traveled to a region with ongoing transmission of Zika virus, but did have sexual contact with a male partner who had recently traveled to the Dominican Republic. The male partner reported exposure to mosquitoes while traveling, but no symptoms consistent with Zika virus infection either before or after returning to the United States. The woman reported no other sex partners during the 14 days before onset of her symptoms and no receipt of blood products or organ transplants.

The couple reported having had condomless vaginal intercourse twice after the man's return from the Dominican Republic and before the woman's symptom onset, approximately 10 days (day 10) and 14 days (day 14) after the man's return. The man also reported that he received fellatio from the woman during their sexual encounter on day 14 . On day 16 ( 2 and 6 days after the episodes of condomless vaginal intercourse) the woman developed symptoms of Zika virus infection, including fever and rash. On day 19 (3 days after symptom onset) she sought medical care; the provider suspected Zika virus infection, and serum and urine specimens were collected. Flavivirus and chikungunya virus tests were performed at the Maryland DHMH Laboratories Administration. Zika virus RNA was detected in urine, but not in serum, by real-time reverse transcription-polymerase chain reaction (rRT-PCR) using a test based on an assay developed at CDC (1). Serum rRT-PCR testing for dengue virus and chikungunya virus was negative. Serologic testing was negative for Zika virus immunoglobulin $\mathrm{M}(\mathrm{IgM})$ antibodies using the CDC Zika IgM antibody capture enzyme-linked immunosorbent assay (Zika MAC-ELISA) and negative for dengue virus and chikungunya virus IgM antibodies using InBios ELISA kits (InBios International, Inc., Seattle, Washington). Confirmatory serologic testing at the CDC Arbovirus Diagnostic Laboratory was equivocal for Zika virus IgM antibodies using the Zika MAC-ELISA. Plaque-reduction neutralization tests (PRNTs) performed at the CDC Arbovirus Diagnostic Laboratory confirmed a recent Zika virus infection. Convalescent serologic testing performed at the Maryland DHMH Laboratories Administration on day 56 (40 days after symptom onset) was equivocal for Zika virus IgM antibodies using the CDC Zika MAC-ELISA and negative for dengue virus and chikungunya virus IgM antibodies using InBios ELISA kits. PRNTs performed at the CDC Arbovirus Diagnostic Laboratory confirmed a recent, unspecified flavivirus infection.

The woman's male sex partner was interviewed on day 26 after his return to the United States. He reported that he had no symptoms consistent with Zika virus infection (i.e., fever, rash, conjunctivitis, or arthralgias) either during his travel or since his return, and he did not have any of the following other symptoms: myalgias, chills, eye pain, oral ulcers, genital ulcers, anal ulcers, hematospermia, hematuria, dysuria, and prostate pain. He reported feeling tired, which he attributed to having recently traveled. Serum, plasma, and urine specimens were collected from him on day 29, at which time he reported no new symptoms. Zika virus rRT-PCR testing performed at the Maryland DHMH Laboratories Administration was negative on serum and plasma and equivocal on urine. Serologic testing was positive for Zika virus IgM antibodies using the CDC Zika MAC-ELISA and positive for dengue virus IgM antibodies using an InBios ELISA kit. PRNTs performed at the CDC Arbovirus Diagnostic Laboratory confirmed a recent, unspecified flavivirus infection. Semen collected on day 31 had no detectable Zika virus RNA by rRT-PCR testing performed at the Maryland DHMH Laboratories Administration.

To date, only one other case has been reported in which a man without symptoms might have sexually transmitted Zika virus to his female partner (2). However, in that reported case, both the man and the woman had traveled to a country with ongoing Zika virus transmission where they were likely exposed to mosquitoes. In that case, although the detection of Zika virus RNA in the woman's serum and urine by rRT-PCR 39 days after return from travel suggested sexual transmission from her male partner, it could not be ruled out that she had been infected from a mosquito bite during travel and had a longer than average incubation period or a prolonged period of viremia. No cases of sexual transmission of Zika virus from an asymptomatic man returning from travel to an area with active Zika transmission to his female sex partner who did 
not travel have been reported. Absence of Zika virus symptoms in persons returning from areas with ongoing Zika virus transmission might not preclude sexual transmission of Zika virus to their sex partners. Ongoing surveillance is needed to determine the risk for sexual transmission of Zika virus infection from asymptomatic persons. The findings in this report indicate that it might be appropriate to consider persons who have condomless sex with partners returning from areas with ongoing Zika virus transmission as exposed to Zika virus, regardless of whether the returning traveler reports symptoms of Zika virus infection. Providers should request Zika virus testing for any patients with illness compatible with Zika virus disease who have had sexual exposure without barrier devices to prevent infections to a partner who traveled to an area with active Zika virus transmission (3). Such patients should also be reported to local or state health departments $(4,5)$.

Current recommendations for the prevention of sexual transmission of Zika virus in returning travelers differ depending on whether the returning traveler is symptomatic and on whether the couple is planning to become pregnant $(3,6)$. Couples in areas without active Zika transmission with circumstances in which one partner traveled to an area with active Zika virus transmission but did not develop symptoms of Zika virus disease should wait at least 8 weeks after the partner who traveled returned from the Zika-affected area before attempting conception, regardless of the sex of the traveler. Men with a diagnosis of Zika virus infection should wait at least 6 months before attempting conception, and women with a diagnosis of Zika virus infection should wait at least 8 weeks before attempting conception. Health care providers should counsel couples that correct and consistent use of condoms reduces the risk for sexually transmitted diseases and discuss the use of the most effective contraceptive methods that can be used correctly and consistently (G). Couples who do not desire pregnancy should consider abstaining from sex or using the most effective contraceptive methods that can be used correctly and consistently in addition to barrier methods, such as condoms, which reduce the risk for sexual transmission of Zika virus and other sexually transmitted infections (3). As more is learned about the incidence and duration of seminal shedding of Zika virus in infected men, recommendations to prevent sexual transmission of Zika virus will be updated if needed.

\footnotetext{
${ }^{1}$ Epidemic Intelligence Service, Division of Scientific Education and Professional Development, CDC; ${ }^{2}$ Prevention and Health Promotion Administration, Maryland Department of Health and Mental Hygiene; ${ }^{3}$ Maryland Department of Health and Mental Hygiene Laboratories Administration; ${ }^{4}$ Baltimore City Health Department, Maryland; ${ }^{5}$ Montgomery County Department of Health and Human Services, Maryland
}

Corresponding author: Richard B. Brooks, richard.brooks@maryland.gov, 410-767-7395.

\section{References}

1. Lanciotti RS, Kosoy OL, Laven JJ, et al. Genetic and serologic properties of Zika virus associated with an epidemic, Yap State, Micronesia, 2007. Emerg Infect Dis 2008;14:1232-9. http://dx.doi.org/10.3201/ eid1408.080287

2. Fréour T, Mirallié S, Hubert B, et al. Sexual transmission of Zika virus in an entirely asymptomatic couple returning from a Zika epidemic area, France, April 2016. Euro Surveill 2016;21:30254. http://dx.doi. org/10.2807/1560-7917.ES.2016.21.23.30254

3. Brooks JT, Friedman A, Kachur RE, LaFlam M, Peters PJ, Jamieson DJ. Update: interim guidance for prevention of sexual transmission of Zika virus-United States, July 2016. MMWR Morb Mortal Wkly Rep 2016;65:745-7. http://dx.doi.org/10.15585/mmwr.mm6529e2

4. Oster AM, Russell K, Stryker JE, et al. Update: interim guidance for prevention of sexual transmission of Zika virus-United States, 2016. MMWR Morb Mortal Wkly Rep 2016;65:323-5. http://dx.doi. org/10.15585/mmwr.mm6512e3

5. Davidson A, Slavinski S, Komoto K, Rakeman J, Weiss D. Suspected female-to-male sexual transmission of Zika virus-New York City, 2016. MMWR Morb Mortal Wkly Rep 2016;65:716-7. http://dx.doi. org/10.15585/mmwr.mm6528e2

6. Petersen EE, Polen KN, Meaney-Delman D, et al. Update: interim guidance for health care providers caring for women of reproductive age with possible Zika virus exposure-United States, 2016. MMWR Morb Mortal Wkly Rep 2016;65:315-22. http://dx.doi.org/10.15585/mmwr. $\mathrm{mm} 6512 \mathrm{e} 2$ 